\title{
Bayesian Optimization Assisted Meal Bolus Decision Based on Gaussian Processes Learning and Risk-Sensitive Control ${ }^{\star}$
}

\author{
Deheng Cai ${ }^{\mathrm{a}}$, Wei Liu ${ }^{\mathrm{b}}$, Linong Ji ${ }^{\mathrm{b}}$, Dawei Shi ${ }^{\mathrm{a}, *}$ \\ ${ }^{a}$ State Key Laboratory of Intelligent Control and Decision of Complex Systems, School of Automation, Beijing Institute of Technology, Beijing, China \\ ${ }^{b}$ Department of Endocrine and Metabolism, Peking University People's Hospital, Beijing, China
}

\begin{abstract}
Effective postprandial glucose control is important to glucose management for subjects with diabetes mellitus. In this work, a 'data-driven meal bolus decision method is proposed without the need of subject-specific glucose management parameters. The postprandial glucose dynamics is learnt using Gaussian process regression. Considering the asymmetric risks of hyper- and hypo'glycemia and the uncertainties in the predicted glucose trajectories, an asymmetric risk-sensitive cost function is designed. Bayesian optimization is utilized to solve the optimization problem, since the gradient of the cost function is unavailable. The proposed approach is evaluated using the 10-adult cohort of the FDA-accepted UVA/Padova T1DM simulator and compared with the standard insulin bolus calculator. For the case of announced meals, the proposed method achieves satisfactory and similar performance in terms of mean glucose and percentage time in $[70,180] \mathrm{mg} / \mathrm{dL}$ without increasing the risk of hypoglycemia. Similar results are observed for the case without the meal information (assuming that the patient follows a consistent diet) and the case of basal rate mismatches. In addition, advisory-mode analysis is performed based on clinical data, which indicates that the method can determine safe and reasonable meal boluses in real clinical settings. The results verify the effectiveness and robustness of the proposed method and indicate the feasibility of achieving improved postprandial glucose regulation through a data-driven optimal control method.
\end{abstract}

Keywords: Meal bolus decision, Gaussian processes, risk-sensitive control, Bayesian optimization.

\section{Introduction}

Diabetes mellitus (DM) is a chronic metabolic disease that is characterized by absolute insulin deficiency (type 1 diabetes (T1D)) or relative lack of insulin secretion and sensitivity (type 2 diabetes (T2D)). Patients with DM tend to suffer from the long term complications, e.g., retinopathy and nephropathy, due to poor blood glucose (BG) managements [1]. Nowadays, external insulin administration through a basal-bolus strategy with multiple daily injections (MDI) or continuous subcutaneous in'sulin infusion (CSII) pump is a popular way among the patients to control BG [2]. With improved modern diabetes technolo'gies, a closed-loop control system for BG regulations, named artificial pancreas (AP), is further developed through integrating the pumps and continuous glucose monitoring (CGM) sensors. An AP automatically delivers insulin to achieve desired BG levels, based on CGM-driven feedback control algorithms $[3,4,5,6]$.

However, for the therapies above, the effective control of postprandial glucose still remains a challenge [7]. At present, the feedforward control action in terms of preprandial insulin

^ This work was supported in part by the National Natural Science Foundation of China under Grant 61973030, and in part by the Peking University People's Hospital Scientific Research Development Funds RDY2019-05.

${ }^{*}$ Corresponding author

Email address: daweishi@bit.edu.cn (Dawei Shi) bolus is widely adopted to counteract the hyperglycemia associated with meal intakes. Specifically, a meal bolus is determined by a bolus calculator according to carbohydrate content of the meal, the BG level and subject-specific settings (carbohydrate ratio $(\mathrm{CR})$ and correction factor $(\mathrm{CF})$ profiles). Multiple efforts have been devoted to improve the performance of the bolus advisors. Considering the repetitive nature of the daily activities of the patient, Owens et al. [8] proposed a Run-to-Run (R2R) algorithm to update the insulin bolus amount and timing daily. In Schiavon et al. [9], a novel optimization method for CR was developed based on a validated index of insulin sensitivity estimated form CGM and CSII data. Combining case-based reasoning $(\mathrm{CBR})$ with $\mathrm{R} 2 \mathrm{R}$, an advanced insulin bolus advisor through adapting $\mathrm{CR}$ and $\mathrm{CF}$ was presented in Herrero et al. [10]. Similarly, Torrent-Fontbona [11] investigated a bolus insulin recommend system based on $\mathrm{CBR}$, but provided a new reuse, revise and retain mechanism to adapt $C R$ and $C F$. Besides $\mathrm{CBR}$, other artificial intelligence techniques including fuzzy logic [12] and reinforcement learning (RL) [13] have been investigated for the bolus advisors. Moreover, several algorithms have also been explored to calculate the required bolus within the framework of an AP. In Turksoy et al. [14], a meal bolus calculation method was developed for unannounced meals based on the Bergman's minimal model and unscented Kalman filter. In Toffanin et al. [15], R2R was implemented in the AP to adapt CR. Shi et al. [16] explored a Bayesian optimization assisted learning framework to adapt CR profiles for the AP, 
using historical CGM and CSII data.

Most of the methods above improve postprandial glucose control through updating $\mathrm{CR}$ and $\mathrm{CF}$ according to the designed mechanisms. These methods do not capture the dynamics of glucose metabolism, and ignore the upcoming postprandial glucose situations in the optimization of bolus dosage, thus leading to potentially sub-optimal glycemic control. With the improved glucose sensor accuracy and accessibility, data-driven optimal control provides a promising way to achieve optimal glycemic control and reduce the burden of optimizing CR and $\mathrm{CF}$. The data-driven optimal control can explicitly exploit the modeled dynamics for the bolus decision by taking account of the preprandial glucose levels, and optimizing the bolus dosage using the predicted postprandial glucose situations. This forms the motivation of our work. To implement the data-driven optimal control for the meal bolus decision, glucose prediction is a critical role. Along this direction, many methods have been explored in the literature [17]. For example, an autoregressive with exogenous input model was presented in RomeroUgaldein et al. [18] to predict interstitial glucose. In Yu et al. [19], four different adaptive filters and a fusion mechanism were proposed for the online glucose concentration predictions. Combining feature ranking with support vector regression or Gaussian processes, Georga et al. [20] investigated the shortterm glucose prediction. To improve long-term glucose prediction, Montaser et al. [21] presented an integrated predicting method based on seasonal local models and fuzzy $c$-means. Different from the predictions above, using the Gaussian process (GP) regression, we provide multi-step predictions (e.g., 2 hours) for the postprandial glucose trajectories corresponding to various preprandial glucose situations, meal boluses and meal information (carbohydrate content), so that the optimization for the meal bolus can be done via the predicted glucose trajectories.

Since GP is a data-efficient and robust modeling method, different approaches have concerned the research of GP-based control. For example, in [22], a framework of probabilistic inference for learning control was proposed based on the GP and applied in real robotics and control tasks. In [23], a GP-based model predictive controller (MPC) was investigated for building energy control and demand response. To enhance effective online learning and control, a risk-sensitive cost was introduced in the MPC with GP models [24], as well as in the RL [25]. Inspired by the work in $[24,25]$, we utilize the predicted information in a risk-sensitive fashion, but construct an asymmetric risk-sensitive cost with the consideration of asymmetric risks in hyper- and hypoglycemia. Finally, based on the designed cost, a constrained stochastic optimization problem is proposed for the meal bolus decision. Since the gradient of the cost cannot be computed analytically, we utilize Bayesian optimization [26] and Monte-Carlo method to solve the optimization problem. For safety reasons, the final solution for the meal bolus enforces an insulin on board (IOB) constraint.

The effectiveness and robustness of the proposed method are evaluated using different in silico protocols on the 10-adult cohort of the US Food and Drug Administration (FDA) accepted Universities of Virginia (UVA)/Padova T1DM simulator, and compared with the standard insulin bolus calculator. For the case of announced meals, the proposed method achieves satisfactory and similar performance for scenarios of nominal basal rates, in terms of mean glucose level and percent time in the safe range, without increasing the risk of hypoglycemia. Similar results are observed for the case without the meal information (assuming that the patient follows a consistent diet) and the scenarios of over/under-estimated basal rates. In addition, advisorymode analysis [27] based on clinical data from a T1DM subject show that the proposed method can determine reasonable meal boluses by explicitly taking account of the preprandial glucose levels in the data-driven optimal control.

\section{Materials and Methods}

The overall structure of the proposed data-driven meal bolus decision method is illustrated in Fig. 1. The method builds on three key components: model learning, asymmetric risksensitive control, and Bayesian optimization.

Model learning is responsible for constructing the postprandial glucose dynamics. Here, the aim is to provide a robust description for postprandial glucose dynamics using GPs. Specifically, fed with the serialized data samples (including preprandial glucose measurements, the corresponding meal information, bolus dosage and postprandial glucose measurements), the GPs are trained offline and then applied online to provide the prediction and the uncertainty estimation of postprandial glucose trajectories. Considering the asymmetric risks of hyperand hypoglycemia and the uncertainties in the predicted glucose trajectories, we develop an asymmetric risk-sensitive cost function to favor safe control actions. Finally, a constrained stochastic optimization problem is formulated for the meal bolus decision based on the designed cost function. Since the gradient of the cost function is unavailable, we solve the optimization problem using Bayesian optimization and Monte-Carlo simulations. To ensure the safety of the method, IOB constraints are also incorporated.

\subsection{Gaussian Processes for Model Learning}

\subsubsection{Gaussian Processes}

A GP assumes a distribution over random functions $f(x)$ : $\mathbb{R}^{n} \rightarrow \mathbb{R}$, such that values of $f$ at any input $x$ have a joint Gaussian distribution $[28,29]$, which is denoted as

$$
f(x) \sim \mathcal{G P}\left(m(x), k\left(x, x^{\prime}\right)\right),
$$

where $m(x)$ and $k\left(x, x^{\prime}\right)$ are the mean function and positive semidefinite covariance function, respectively, which have the form:

$$
\begin{aligned}
m(x) & =\mathbb{E}_{f}[f(x)], \\
k\left(x, x^{\prime}\right) & =\operatorname{cov}_{f}\left[f(x), f\left(x^{\prime}\right)\right], x, x^{\prime} \in \mathbb{R}^{n} .
\end{aligned}
$$

In GPs, we can encode our prior knowledge about the process by designing corresponding prior mean and covariance functions, which are parameterized by the parameter vector $\theta_{f}$. Considering the noisy observations $y$ of $f$ with the form of 


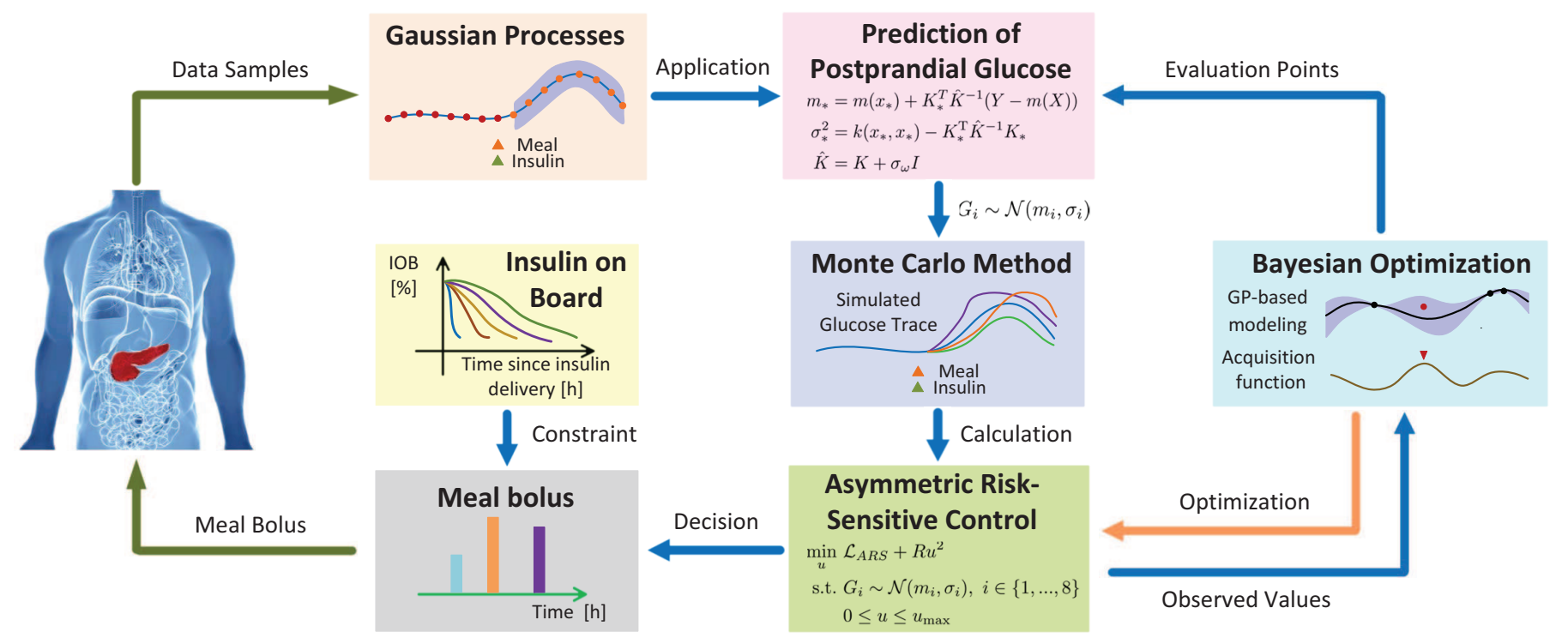

Figure 1: Schematic of the proposed method.

$y=f(x)+\omega$, where $\omega \sim \mathcal{N}\left(0, \sigma_{\omega}^{2}\right)$ is a white Gaussian noise, the covariance function of $y$ has the form:

$$
k_{y}\left(x, x^{\prime}\right)=k\left(x, x^{\prime}\right)+\sigma_{\omega}^{2} \delta\left(x, x^{\prime}\right),
$$

where $\delta\left(x, x^{\prime}\right)$ is the Kronecker delta function which is one if and only if $x=x^{\prime}$ and zero otherwise. Given $N$ training inputs $X=\left[x_{1}, x_{2}, \ldots, x_{N}\right]^{\top}$ and the corresponding observations $Y=\left[y_{1}, y_{2}, \ldots, y_{N}\right]^{\top}$, the posterior GP hyper-parameters $\theta=\left[\theta_{f}, \sigma_{\omega}\right]$ are learned by maximizing the log-marginal likelihood: $\arg \max _{\theta} \log (p(Y \mid X, \theta))$ [29].

With the determined hyper-parameters, the GP can infer the posterior distribution of $y_{*}$ corresponding to a new input $x_{*}$ : $y_{*} \sim \mathcal{N}\left(m_{*}, \sigma_{*}^{2}\right)$ with

$$
\begin{aligned}
m_{*} & =m\left(x_{*}\right)+K_{*}^{\top}\left(K+\sigma_{\omega} I\right)^{-1}(Y-m(X)), \\
\sigma_{*}^{2} & =k\left(x_{*}, x_{*}\right)-K_{*}^{\top}\left(K+\sigma_{\omega} I\right)^{-1} K_{*},
\end{aligned}
$$

where $K_{*}=\left[k\left(x_{*}, x_{1}\right), \ldots, k\left(x_{*}, x_{N}\right)\right]^{\top}$ and $K$ is the covariance matrix with elements $K_{i j}=k\left(x_{i}, x_{j}\right)$. As shown in (5) and (6), for a new input, a GP can provide a determined prediction via predictive mean, c.f., (5), along with an estimate of uncertainty or confidence in the prediction via the predictive variance, c.f., (6).

\subsubsection{Postprandial Glucose Prediction}

Physiologically-based compartmental models are commonly utilized to describe glucose dynamics using first order differential equations $[30,31,32]$; however, the parameter identification of these models is time-consuming and sometimes is even impossible with incomplete information, e.g., when only the CGM data is available. Here, we utilize GPs to perform robust modeling for postprandial glucose dynamics with incomplete and noisy information. Specifically, by feeding autoregressive, or time-delayed, input and output signals back to the model as regressors, the GPs can be used for modeling nonlinear dynamical control systems [22, 23].
To do this, the postprandial glucose (PG) dynamics is described in a multistep form using autoregressive models, and each step is separately represented by a nonlinear function with additive noise, which has the form:

$$
\begin{aligned}
P_{t+1} & =f_{t}\left(z_{t}\right)+w_{t}, \\
z_{t} & =\left[P_{t-l}, \ldots, P_{t}, u, d\right]^{\top} ; \\
P_{t+2} & =f_{t+1}\left(z_{t+1}\right)+w_{t+1}, \\
z_{t+1} & =\left[P_{t-l+1}, \ldots, P_{t+1}, u, d\right]^{\top} ; \\
& \ldots \\
P_{t+n} & =f_{t+n-1}\left(z_{t+n-1}\right)+w_{t+n-1}, \\
z_{t+n-1} & =\left[P_{t+n-1-l}, \ldots, P_{t+n-1}, u, d\right]^{\top},
\end{aligned}
$$

where $t$ denotes the time of the meal intake, $w$ is a white Gaussian noise, $P$ is the glucose measurement, and $l$ is the lag for autoregressive outputs; $u$ is the meal bolus, and $d$ is the carbohydrate intake. To convey the most information of glucose situations, the lag of $l=7$ and the sampling period of $T=15 \mathrm{~min}$ are considered. This corresponds to the lag of 2 hours. Correspondingly, we take $n=8$; since the sampling period is $15 \mathrm{~min}$, this corresponds to the duration of 2 hours.

The utilization of GPs is divided into two stages: offline modeling and online prediction. In the offline modeling stage, based on (7), the GPs are separately used to model the PG dynamics in each step following a similar way. For example, for the time step $t+1$, we use $z_{t}=\left[P_{t-7}, \ldots, P_{t}, u, d\right]^{\top}$ as training inputs, and the differences $\Delta P_{t}=P_{t+1}-P_{t}$ as training targets to reduce the prediction uncertainty [22]. A linear mean function and a commonly-used covariance function known as squared exponential (SE) covariance kernel are considered:

$$
\begin{aligned}
m\left(z_{t}\right) & =a^{\top} z_{t}+b \\
k_{z}\left(z_{t}, z_{t}^{\prime}\right) & =\sigma_{f}^{2} \exp \left(-\frac{1}{2}\left(z_{t}-z_{t}^{\prime}\right)^{\top} \Omega^{-1}\left(z_{t}-z_{t}^{\prime}\right)\right)+\delta\left(z_{t}, z_{t}^{\prime}\right) \sigma_{\omega}^{2},
\end{aligned}
$$


where $\Omega=\operatorname{diag}\left\{\left[l_{1}^{2}, l_{2}^{2}, \ldots, l_{10}^{2}\right]\right\}, \sigma_{f}^{2}$ denotes the signal variance, and the characteristic length scales for input space $l_{1}, l_{2}, \ldots, l_{10}$ describe the smoothness of the function. Note that $u$ and $d$ stay the same for all steps. Moreover, to highlight the effects of $u$ and $d$ on the glucose regulation, the glucose measurements in the training inputs for each step are separately normalized into $[0,1]$ using min-max normalization.

In the online prediction stage, given a bolus dosage and known carbohydrate amount intakes, by iteratively feeding back the predictive mean of previous step into the input for the prediction, we can obtain the prediction for each step using corresponding trained GPs. This prediction corresponds to the difference between the current step and previous step. We then add this prediction with the predicted mean of the previous step to determine the final prediction in current step. Uniting the predictions for the all steps, the GPs are able to provide 8-step predictions for the PG trajectories that correspond to the corresponding preprandial glcucose situations, carbohydrate intakes and meal boluses. Note that when the eating habit of a subject is approximately consistent in terms of timing and sizes of meal intake, we can approximate the effect of similar food intake as an invariant disturbance and the meal size information can be optional for postprandial glucose prediction, utilizing the robust prediction ability of the GPs; this allows the design of a bolus decision algorithm without meal announcements.

\subsection{Asymmetric Risk-Sensitive Control}

\subsubsection{Asymmetric Risk-Sensitive Cost}

The glucose control problem is highly asymmetric in the sense that consequences of hypoglycemia are immediate and more detrimental in comparison with those of (temporary) hyperglycemia; therefore, we try to correct the postprandial hyperglycemia while taking extra care of hypoglycemia. One feasible method to address this issue is to construct asymmetric cost functions [33, 34]. Inspired by the work in [34], we penalize deviations above and below the target asymmetrically; in addition, considering the uncertainties in postprandial glucose prediction, the cost function is built in the risk-sensitive (RS) framework.

To do this, we denote the 8 -step predictions provided by the GPs as $G_{i} \sim \mathcal{N}\left(m_{i}, \sigma_{i}^{2}\right), i \in\{1,2, \ldots, 8\}$, respectively, and collect them as a vector state $G=\left[G_{1}, G_{2}, \ldots, G_{8}\right]^{\top}$, which describes the probability distribution of postprandial glucose trajectories. According to the principle of risk-sensitive analysis [35], an asymmetric RS cost is designed as follows:

$$
\begin{aligned}
\mathcal{L}_{A R S}=-\frac{2}{\gamma} \log \mathbb{E} & {\left[\operatorname { e x p } \left(-\frac{\gamma}{2}\left(G-G_{r}\right)_{+}^{\top} Q^{+}\left(G-G_{r}\right)_{+}\right.\right.} \\
& \left.\left.-\frac{\gamma}{2}\left(G-G_{r}\right)_{-}^{\top} Q^{-}\left(G-G_{r}\right)_{-}\right)\right],
\end{aligned}
$$

where

$$
\begin{array}{r}
\left(G-G_{r}\right)_{+}=\left[\left(G_{1}-G_{r 1}\right) \mathbf{1}\left(G_{1}-G_{r 1} \geq 0\right), \ldots,\right. \\
\left.\left(G_{8}-G_{r 8}\right) \mathbf{1}\left(G_{8}-G_{r 8} \geq 0\right)\right]^{\top}, \\
\left(G-G_{r}\right)_{-}=\left[\left(G_{1}-G_{r 1}\right) \mathbf{1}\left(G_{1}-G_{r 1}<0\right), \ldots,\right. \\
\left.\left(G_{8}-G_{r 8}\right) \mathbf{1}\left(G_{8}-G_{r 8}<0\right)\right]^{\top},
\end{array}
$$

and $\mathbf{1}(\cdot)$ denotes the indicator function; $G_{r}$ is the target for the postprandial glucose management; $Q^{+}$is a positive penalty matrix for the glucose excursions above the target; $Q^{-}$is a negative penalty matrix for the glucose excursions below the target; $\gamma<0$ is a risk sensitivity parameter that determines the system's attitude towards uncertainty [24, 25]. With the risksensitive cost function, the optimizer is able to fully exploit the experience learned from the historical data while keeping its own decision-making ability.

As for the design of asymmetric penalty, $Q^{+}$is designed as a constant diagonal matrix. Based on the designed $Q^{+}$, the diagonal elements of $Q^{-}$are devised to increase exponentially with the increase of the absolute deviation from target while being restricted by upper and lower bounds. Specifically, the $i$ th diagonal element of $Q^{-}$has the form of

$$
Q_{i}^{-}:=Q_{i}^{+}\left(\frac{c_{1}}{1+\exp \left\{\alpha\left(\beta-\left|G_{i}-G_{r i}\right|\right)\right\}}+c_{2}\right),
$$

where $Q_{i}^{+}$is the $i$ th diagonal element of $Q^{+} ; \Gamma:=\left[\alpha, \beta, c_{1}, c_{2}\right]$ is a quadruple determines the penalty intensity, which is designed same for the all diagonal elements. The lower and upper bounds are determined by $c_{2}$ and $c_{1}+c_{2}$, respectively, and the rate of increase is controlled by $\alpha$. The parameter design will be discussed in Section 3 .

Based on the above design, the quadratic penalty using constant $Q^{+}$is scaled on the excursions above the target to maintain a reasonable but active response to hyperglycemia. Comparatively, a quadratic penalty with exponentially weighted coefficients is applied to the glucose excursions below the target to have a reasonably conservative response to the glucose excursions near the target, while maintaining the ability to respond quickly to larger glucose excursions and safely compensate for hypoglycemia.

\subsubsection{GP-Based Asymmetric Risk-Sensitive Control}

Given the designed asymmetric risk-sensitive cost $\mathcal{L}_{A R S}$ in (10), the GP-based asymmetric risk-sensitive control for the meal bolus decision is formulated as the following constrained stochastic optimization problem,

$$
\begin{aligned}
& \min _{u} \mathcal{L}(u):=\mathcal{L}_{A R S}+R u^{2}, \\
& \text { s.t. } G_{i} \sim \mathcal{N}\left(m_{i}, \sigma_{i}\right), i \in\{1, \ldots, 8\} \\
& \quad 0 \leq u \leq u_{\max },
\end{aligned}
$$

where $u$ is the meal bolus to be optimized, $R$ is the input weighting to compromise the asymmetric RS cost and the actual needed bolus dosage, $m_{i}$ and $\sigma_{i}$ are parameters provided by the GPs.

\subsection{Bayesian Optimization for Meal Bolus Decision}

Since the gradient of the cost function cannot be obtained analytically, Bayesian Optimization (BO) [26] is employed to solve the above constrained stochastic optimization problem. 


\subsubsection{Model Learning for the Cost Function}

Here, we utilize a new GP to construct an approximation of a complex map from the decision variable $u$ to the cost function value $\mathcal{L}(u)$ in (14). Specifically, we consider a prior zero mean function and the SE covariance function in (9) with a scalar input. As discussed in Section 2.1, given the set of $N$ past observations $\mathcal{D}_{1: N}=\left\{u_{1: N}, \mathcal{L}\left(u_{1: N}\right)\right\}$, the GP is trained and then applied to predict the cost function value for a candidate meal bolus $u_{*}$ according to (5)-(6). The prediction is denoted as $\hat{\mathcal{L}}\left(u_{*}\right)$ and will be utilized to construct the acquisition function (see Section 2.3.2).

Note that the value of the cost function corresponding to the decision variable is estimated by Monte-Carlo simulations. Specifically, given a bolus dosage, we generate 1000 samples for the postprandial glucose trajectory based on the joint Gaussian distribution provided by the GP in Section 2.1. The average cost for these samples is further calculated, which is then regarded as the observed value of the cost corresponding to the bolus dosage.

\subsubsection{Acquisition Function}

As a critical ingredient of the $\mathrm{BO}$, the acquisition function guides the optimization by determining the optimum candidate point for the next evaluation. Specifically, utilizing the prediction information offered by the model learning phase, the acquisition function is constructed to determine the candidate point by maintain a trade-off exploration of the search space and exploitation of current promising areas. Up to now, there are rich literature concerning the acquisition function design [26], where several structures have been developed, e.g., probability of improvement, expected improvement, upper confidence bound, and entropy search. In this work, the expected improvement is considered.

Compared with probability of improvement, the expected improvement (EI) acquisition function [36] also incorporates the amount of improvement in selecting the candidate points. Specifically, to minimize the cost in (14), the improvement function of the EI acquisition function is defined as

$$
I\left(u_{*}\right):=\left(\mathcal{L}_{\min }^{p}-\hat{\mathcal{L}}\left(u_{*}\right)\right) \mathbf{1}\left(\mathcal{L}_{\min }^{p}>\hat{\mathcal{L}}\left(u_{*}\right)\right),
$$

where $\mathcal{L}_{\min }^{p}$ denotes the minimum observed value of the cost so far, $\hat{\mathcal{L}}\left(u_{*}\right) \sim \mathcal{N}\left(m\left(u_{*}\right), \sigma^{2}\left(u_{*}\right)\right)$ is the prediction at candidate point $u_{*}$. Note that the term $\mathcal{L}_{\text {min }}^{p}-\hat{\mathcal{L}}\left(u_{*}\right)$ represents the amount of improvement, and the other term denotes the probability of that improvement. Based on the improvement function, the EI acquisition function is further defined as

$$
\alpha_{E I}\left(u_{*}\right):=\mathbb{E}\left(I\left(u_{*}\right)\right) .
$$

By calculating the expectation in (18), we have

$$
\begin{aligned}
& \alpha_{E I}\left(u_{*}\right)= \\
& \begin{cases}\left(\mathcal{L}_{\min }^{p}-m\left(u_{*}\right)\right) \Phi(U)+\sigma\left(u_{*}\right) \phi(U), & \text { if } m\left(u_{*}\right)>0, \\
0, & \text { otherwise, }\end{cases}
\end{aligned}
$$

where

$$
U=\frac{\mathcal{L}_{\min }^{p}-m\left(u_{*}\right)}{\sigma\left(u_{*}\right)},
$$

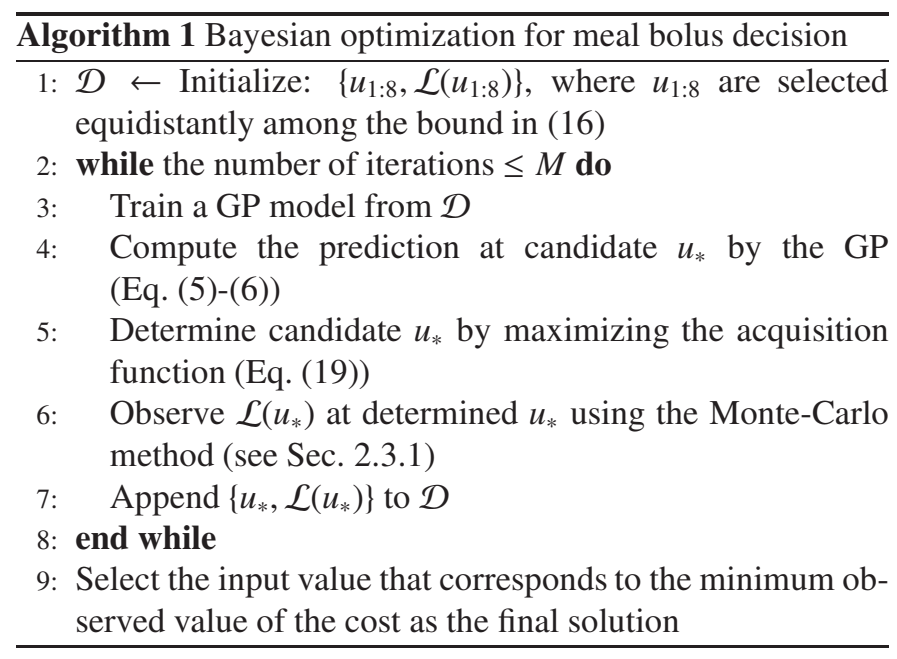

and $\Phi(\cdot)$ is the standard normal cumulative distribution function, and $\phi(\cdot)$ denotes the standard normal probability density function. The candidate point for the next evaluation is determined as the one that maximizes $\alpha_{E I}$.

The $\mathrm{BO}$ algorithm that solves the optimization problem in (14)-(16) is summarized in Algorithm 1. After $M$ sequential operations of the $\mathrm{BO}$, the final solution $\widetilde{u}_{b}$ is determined. For safety concern, an IOB constraint [37] is enforced to prevent overbolus based on insulin delivery history. Denoting the IOB constraint as $u_{\mathrm{IOB}}$, the final meal bolus is determined as

$$
u_{b}=\widetilde{u}_{b}-u_{\mathrm{IOB}} .
$$

\section{Data collection and Parameter design}

The parameters of the proposed method are designed and evaluated using the UVA/Padova T1DM metabolic simulator [38]. The "average patient" of the simulator is selected to perform the parameter design, and the obtained parameters are further evaluated using the 10 virtual adult patients.

\subsection{Data Collection}

Before the parameter design and evaluation, the data samples needed for the PG model learning are collected for each patient. The samples are collected under the situation where the in silico patients combine basal insulin and bolus insulin to control BG. To do this, a protocol with nominal basal rate and announced meals are designed for the patients. The protocol starts from 5:00 on day one and lasts one week (7 days). Considering the daily activities of diabetic patients tend to form similar patterns, e.g., with respect to meal timing and meal amount, but to mimic lifestyle disturbances, we assume the patients take breakfast, lunch, and dinner with normally distributed meal sizes (with means and standard deviations equal to $[50,75,75] \mathrm{g}$ and $[3$, 4 , 4] g CHO) and meal times uniformly distributed in [07:00, 09:00], [11:00, 13:00], and [18:00, 20:00], respectively. The meals are all announced but the meal boluses are calculated with potentially inappropriate $\mathrm{CR}$, such that the variation of the bolus is uniformly distributed in $[-30 \%,+30 \%]$. With the generated data by the protocol, we totally collect 7 samples for 
Table 1: Parameters for the proposed method

\begin{tabular}{ll}
\hline Variables & Value \\
\hline$\gamma$ & -2 \\
$R$ & 4 \\
$u_{\max }$ & 15 \\
$M$ & 25 \\
$Q^{+}$ & $\operatorname{diag}\{[0.01, \cdots, 0.01,0.02,0.02]\}$ \\
$\Gamma$ & {$[1,10,5,1]$} \\
$G_{r}$ & {$[100,120,140,160,160,150,140,140]^{\top}$} \\
\hline
\end{tabular}

the breakfast, lunch and dinner, respectively. Each sample includes preprandial glucose measurements, corresponding bolus dosage, carbohydrate amount, and postprandial glucose measurements. As the consumed $\mathrm{CHO}$ for lunch and dinner are similar but different from the breakfast, we construct the same PG model for both lunch and dinner, and build a separate model for the breakfast using corresponding samples, although it is also possible to build separate models for breakfast, lunch and dinner.

\subsection{Parameter Design}

Using the collected samples, the GPs are trained offline to provide PG predictions for the online control of the method. In the online control, the parameter design and its evaluation are performed. In the parameter design phase, a 12-hour in silico protocol starting from 6:00 is employed, where breakfast (50 $\mathrm{g} \mathrm{CHO}$ ), lunch (75 g CHO) are consumed at 8:00 and 12:00, respectively.

The parameters are designed using a trial-and-error approach based on the glucose data obtained from the "average patient", such that satisfactory postprandial regulation performance in terms of average glucose, percent time in $[70,180] \mathrm{mg} / \mathrm{dL}$, and percent time below $70 \mathrm{mg} / \mathrm{dL}$ can be achieved. The obtained parameters are summarized in Table 1. In the evaluation phase, different scenarios are designed to evaluate the performance of the proposed method with the obtained parameters. The results are reported in Section 4.

\section{Performance Analysis}

As mentioned above, the proposed method is evaluated on the 10-adult cohort of the UVA/Padova T1DM simulator. Besides, the advisory-mode analysis [27] is performed for the method based on the clinical data from a particular T1DM subject who undertook the MDI therapy.

For the in silico evaluations, two protocols are designed to perform the comparison of the proposed method and the standard insulin bolus calculator (denoted as "Control"), which has the form:

$$
u_{\text {bolus }}=\frac{\mathrm{CHO}}{\mathrm{CR}}+\frac{G_{c}-G_{s p}}{\mathrm{CF}}-u_{\mathrm{IOB}},
$$

where $G_{c}$ is the current glucose level (mg/dL); $G_{s p}$ is the glucose set-point (mg/dL), selected as 140 in this comparison. One of the designed protocol (denoted as "Protocol A") begins at
5:00 on day 1 and lasts two days (48 hours) where breakfast (55 $\mathrm{g}$ and $45 \mathrm{~g} \mathrm{CHO})$, lunch (65 g and $85 \mathrm{~g} \mathrm{CHO}$ ) and dinner (85 $\mathrm{g}$ and $65 \mathrm{~g} \mathrm{CHO}$ ) are consumed at 8:00, 12:00 and 18:00 for two days, respectively. The other protocol (denoted as "Protocol B") begins at 5:00 and lasts one day (24 hours), including breakfast, lunch and dinner with $45 \mathrm{~g}, 85 \mathrm{~g}$, and $65 \mathrm{~g}$ of CHO content at 8:00, 12:00 and 18:00, respectively. Based on protocol A, in silico experiments are performed on the whole 10 -adult cohort with nominal basal rates using additive CGM noises for scenario (not) utilizing the meal information for the prediction, thus a total of 10 simulations are performed for each scenario and each method. The statistical results are summarized in Table 2, and the comparison performance in terms of glucose regulation and meal bolus is illustrated in Fig. 2, where the $5 \%, 25 \%, 75 \%$ and $95 \%$ quartile curves together with the median curves are presented. Besides, to illustrate the robust decision-making ability of the proposed method, in silico evaluations without meal information for scenarios of basalrate mismatches (110\% and $80 \%$ of the nominal basal rate) are performed on the same cohort using protocol B, respectively. The results are presented in Table 3 and Fig. 3, and the corresponding discussions for the both protocol are provided in Section 4.1.

The advisory-mode analysis [27] allows the comparisons with insulin recommendations made by clinicians, through feeding the identical glucose data obtained in the clinical trial to the system. Here, by feeding the historical preprandial glucose data to the proposed method, the corresponding meal boluses are determined and compared with the ones following the clinician's advice. The obtained meal boluses have no causal impact on historical data and are only for the comparison. The collection of the historical clinical data are presented in Fig. 4, and the comparison performance are illustrated in Fig. 5. The corresponding discussions are provided in Section 4.2.

\subsection{In Silico Performance Evaluation}

In this subsection, using Protocol A, the performance of the proposed method are evaluated for the cases with the meal information (IM) and without the meal information (NM), respectively. From Table 2, for the both cases, the proposed method achieves comparable glucose regulation performance in comparison with the standard insulin bolus calculator, which is equipped with the well-designed CR and CF. This is reflected in percent time in the euglycemic range of $70-180 \mathrm{mg} / \mathrm{dL}(96.6 \%$ vs. $95.8 \%, p=0.627$ for IM; $95.4 \%$ vs. $95.8 \%, p=0.264$ for $\mathrm{NM})$, mean glucose $(126.7 \mathrm{mg} / \mathrm{dl}$ vs. $132.8 \mathrm{mg} / \mathrm{dL}, p=0.037$ for IM; $131.0 \mathrm{mg} / \mathrm{dL}$ vs. $132.8 \mathrm{mg} / \mathrm{dL}, p=0.084$ for $\mathrm{NM})$, percent time $>250 \mathrm{mg} / \mathrm{dL}(0.0 \%$ vs. $0.0 \%, p=1.000$ for IM; $0.0 \%$ vs. $0.0 \%, p=1.000$ for NM) and glucose standard deviation (SD) (25.9 vs. 26.2, $p=1.000$ for IM; 27.0 vs. $26.2, p=0.020$ for NM). No increase in the risk of hypoglycemia is observed (percent time $<70 \mathrm{mg} / \mathrm{dL}, 0.0 \%$ vs. $0.0 \%$, $p=0.500$ for IM; $0.0 \%$ vs. $0.0 \%, p=0.500$ for NM). These results show the method is robust to the meal information if the $\mathrm{CHO}$ amount intakes are similar to the standard amount used for the model learning, and also illustrate the learning ability of proposed method, as the samples for the model learning are 
Table 2: Comparison results with nominal basal rate

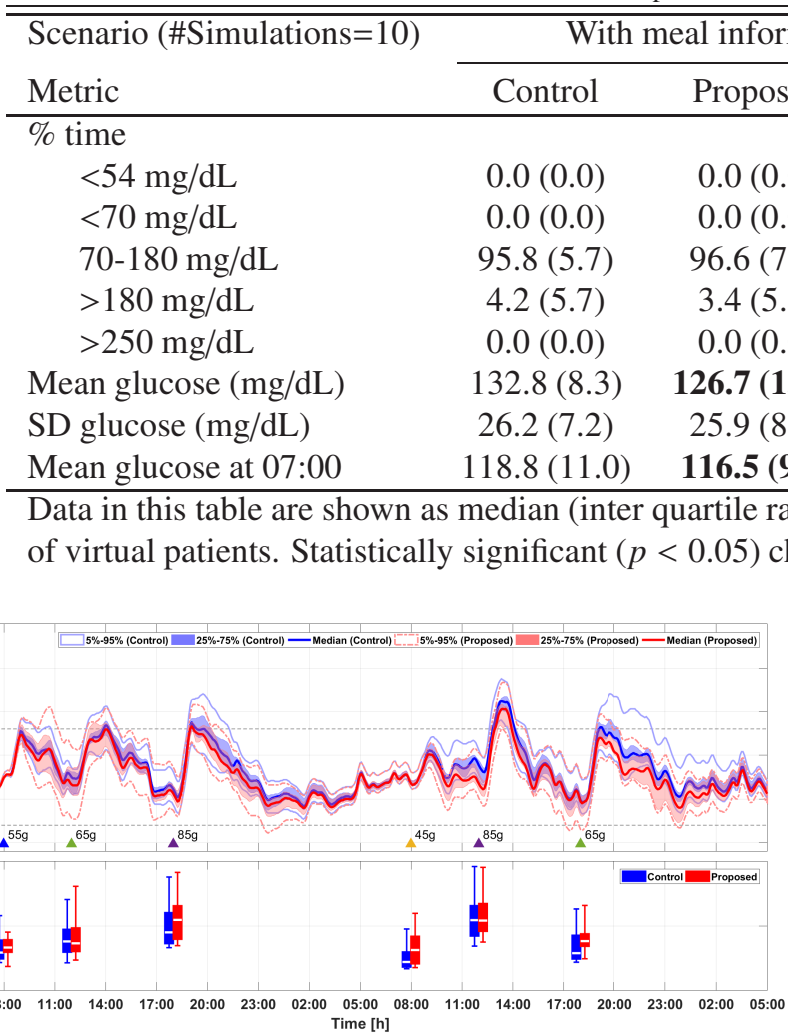

(a) With meal information

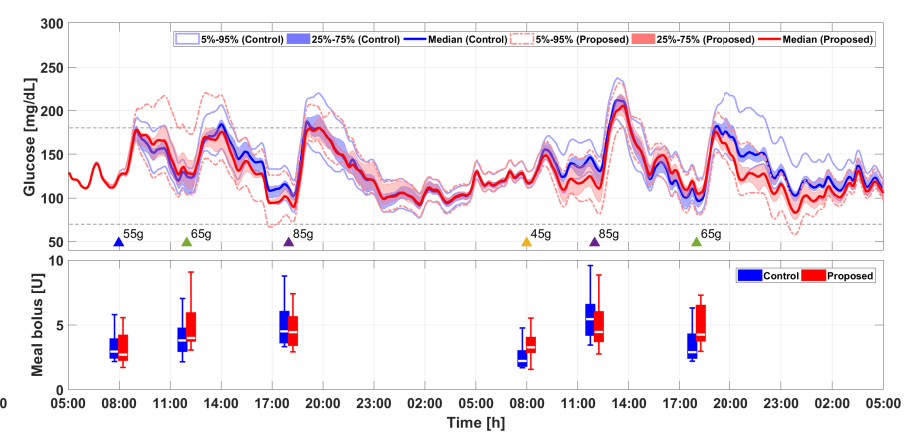

(b) Without meal information

Figure 2: Performance comparison with nominal basal rate in terms of glucose regulation and meal bolus. Yellow, blue, green and purple triangles denote meals of $45 \mathrm{~g}, 55 \mathrm{~g}, 65 \mathrm{~g}$ and $85 \mathrm{~g} \mathrm{CHO}$, respectively.

Table 3: Comparison results with under/over-estimated basal rate

\begin{tabular}{|c|c|c|c|c|c|c|}
\hline \multirow{2}{*}{$\begin{array}{l}\text { Scenario (\#Simulations }=10 \text { ) } \\
\text { Metric }\end{array}$} & \multicolumn{3}{|c|}{$80 \%$ of the nominal basal rate } & \multicolumn{3}{|c|}{$110 \%$ of the nominal basal rate } \\
\hline & Control & Proposed & $p$ value & Control & Proposed & $p$ value \\
\hline \multicolumn{7}{|l|}{$\%$ time } \\
\hline$<54 \mathrm{mg} / \mathrm{dL}$ & $0.0(0.0)$ & $0.0(0.0)$ & 1.000 & $0.0(0.0)$ & $0.0(0.0)$ & 1.000 \\
\hline$<70 \mathrm{mg} / \mathrm{dL}$ & $0.0(0.0)$ & $0.0(0.0)$ & 1.000 & $0.0(0.0)$ & $0.0(3.1)$ & 0.625 \\
\hline $70-180 \mathrm{mg} / \mathrm{dL}$ & $87.8(3.5)$ & $87.7(8.0)$ & 0.389 & $96.5(3.8)$ & $95.0(9.7)$ & 0.586 \\
\hline$>180 \mathrm{mg} / \mathrm{dL}$ & $12.2(3.5)$ & $12.3(8.0)$ & 0.389 & $3.5(4.9)$ & $4.7(7.3)$ & 0.945 \\
\hline$>250 \mathrm{mg} / \mathrm{dL}$ & $0.0(0.0)$ & $0.0(0.0)$ & 1.000 & $0.0(0.0)$ & $0.0(0.0)$ & 1.000 \\
\hline Mean glucose (mg/dL) & $151.2(4.9)$ & $146.6(10.4)$ & 0.131 & $120.9(6.9)$ & $119.0(7.6)$ & 0.064 \\
\hline SD glucose $(\mathrm{mg} / \mathrm{dL})$ & $24.7(5.9)$ & $26.8(5.9)$ & 0.131 & $28.8(4.8)$ & $32.0(8.9)$ & 0.275 \\
\hline Mean glucose at 07:00 & $151.0(21.0)$ & $145.0(27.0)$ & 0.047 & $93.0(11.0)$ & $93.0(12.0)$ & 0.559 \\
\hline
\end{tabular}

Data in this table are shown as median (inter quartile range), and $p$ values are calculated based on the number of virtual patients. Statistically significant $(p<0.05)$ changes are highlighted in bold.

collected with the inappropriate $\mathrm{CR}$. The discussions of control performance are consistent with the quartile curves in Fig. 2. Besides, from the quartile curves of meal bolus in Fig. 2, we observe that compared with the case of NM, the method in the case of IM tends to increase (decrease) the meal bolus for the known large (small) CHO. This implies that when the accurate meal information are available, the method can react reasonably to the $\mathrm{CHO}$ amount, but this will increase the burden of data collection for model learning in return.

Using Protocol B, we also perform additional tests considering realistic scenarios of under/over-estimated basal rates with/without meal information to evaluate the robustness of the proposed method. The results are also compared with those obtained for the standard insulin bolus calculator. Since similar results are observed for both cases, here we only present the results of the case without meal information. From Table 3 and 


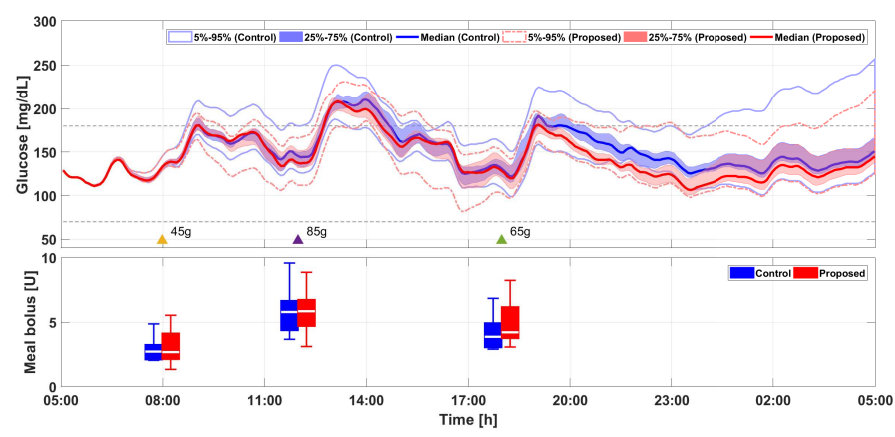

(a) Under-estimated basal rates

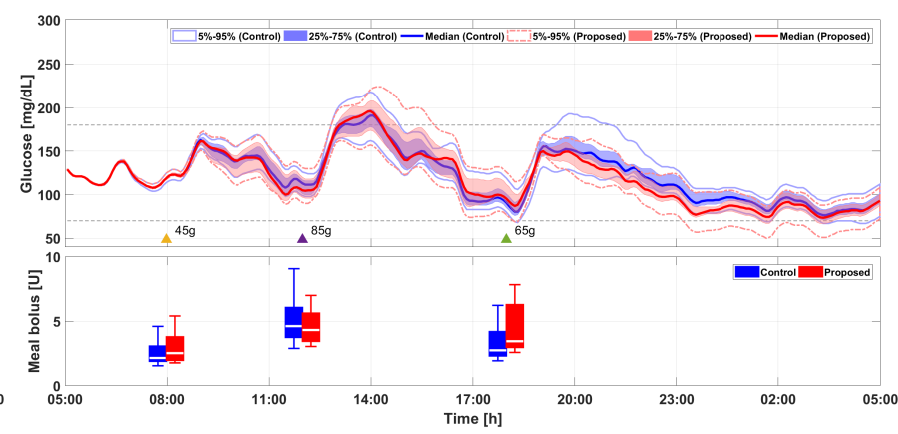

(b) Over-estimated basal rates

Figure 3: Performance comparison for the scenario of under-/over-estimated basal rates.

Fig. 3, it is observed that for the scenario of under-estimated basal rate, the proposed method tends to increase the meal bolus for the elevated preprandial glucose levels, and achieves similar performance in terms of percent time in $[70,180] \mathrm{mg} / \mathrm{dL}$ (87.7\% vs. $87.8 \%, p=0.389)$ and mean glucose $(146.6 \mathrm{mg} / \mathrm{dL}$ vs. $151.2 \mathrm{mg} / \mathrm{dL}, p=0.131)$ without causing risk of hypoglycemia (percent time below $70 \mathrm{mg} / \mathrm{dL}, 0.0 \%$ vs. $0.0 \%$, $p=1.000)$. Similar results are observed for the scenario of over-estimated basal rate, which are reflected in percent time in $[70,180] \mathrm{mg} / \mathrm{dL}(95.0 \%$ vs. $96.5 \%, p=0.586)$ and mean glucose $(119.0 \mathrm{mg} / \mathrm{dL}$ vs. $120.9 \mathrm{mg} / \mathrm{dL}, p=0.064)$ without causing risk of hypoglycemia $(0.0 \%$ vs. $0.0 \%, p=0.625)$. These results further illustrate the robust decision-making ability of the method for the extreme preprandial glucose situations.

Finally, we would like to note that the extremely satisfactory glucose control performance obtained by the proposed approach and the standard bolus calculator is partially attributed to the simulator dynamics and should not be over-emphasized, as we observe that the time-in-range achieved using the standard bolus calculator (using the $\mathrm{CR}$ and $\mathrm{CF}$ values provided by the simulator) goes beyond $95 \%$ in Table 2 . The aim of presenting the in silico evaluation results, however, is to compare the proposed data-driven method with the standard approach that is built on CR and CF information.

\subsection{Advisory-Mode Comparisons Using Clinical Data}

In this subsection, the historical clinical data from a T1DM subject who undertook the MDI therapy are utilized to evaluate the performance of the proposed method. Flash glucose monitoring (FGM) was worn to collect the glucose measurements. The data for the seven days (see Fig. 4) were collected at hospital, where the patient was managed to have a consistent diet for every day, in terms of similar meal timing and meal intakes, and the corresponding meal boluses were determined by the clinicians. The study was approved by institutional review board at Peking University People's Hospital and written informed consent of the participant was obtained. Since the meal intakes are almost identical for every day, we use the data of the first five days to model the PG dynamics for the breakfast and lunchdinner, respectively, without the meal information. At last, the performance of the proposed method equipped with the trained GPs is evaluated by feeding the data for the next two days.
From Fig. 5, compared with the fixed meal boluses (denoted as blue bars) following the clinician's advice, the proposed method can determine reasonable meal boluses (denoted as red bars) according to the preprandial glucose levels. We observe that the method would suggest additional 1-2 units of the insulin bolus for the lunch or dinner of the two days due to the elevated preprandial glucose level. This reasonable increase bolus would help reduce the later happened hyperglycemia. Besides, the breakfast meal boluses are the same as those determined by the clinicians; observing that the historical breakfast boluses are almost all identical, this indicates that the risk-sensitive control mechanism tends to maintain the decisions in the database to ensure safety when the risk of taking a different value is not clear.

\section{Conclusion}

In this work, a GP-based asymmetric risk-sensitive (ARS) control method is proposed for the personalized meal bolus decision. With the formulation of the ARS cost function, the method is capable to apply the experience learned form the samples, while keeping own decision-making ability, e.g., taking extra care of the hyperglycemia and increasing (decreasing) the meal bolus for the elevated (lowered) preprandial glucose levels. Besides, the method is robust to the meal variability within a tolerable range, which reduces the burdens of estimating the $\mathrm{CHO}$ amount for each meal. The effectiveness and robustness of the controller are evaluated using the 10adult cohort of the UVA/Padova simulator through comparisons with the standard insulin bolus calculator. Also, advisory-mode analysis is performed based on the clinical data from a T1DM subject. For future work, since the model learning is relied on the offline samples, an adaptive PG model will be developed for the method to take the long-term variations of the physiological dynamics into account.

\section{Acknowledgment}

Access to the distributed version of the University of Virginia (UVA)/Padova metabolic simulator for research purposes is acknowledged. 

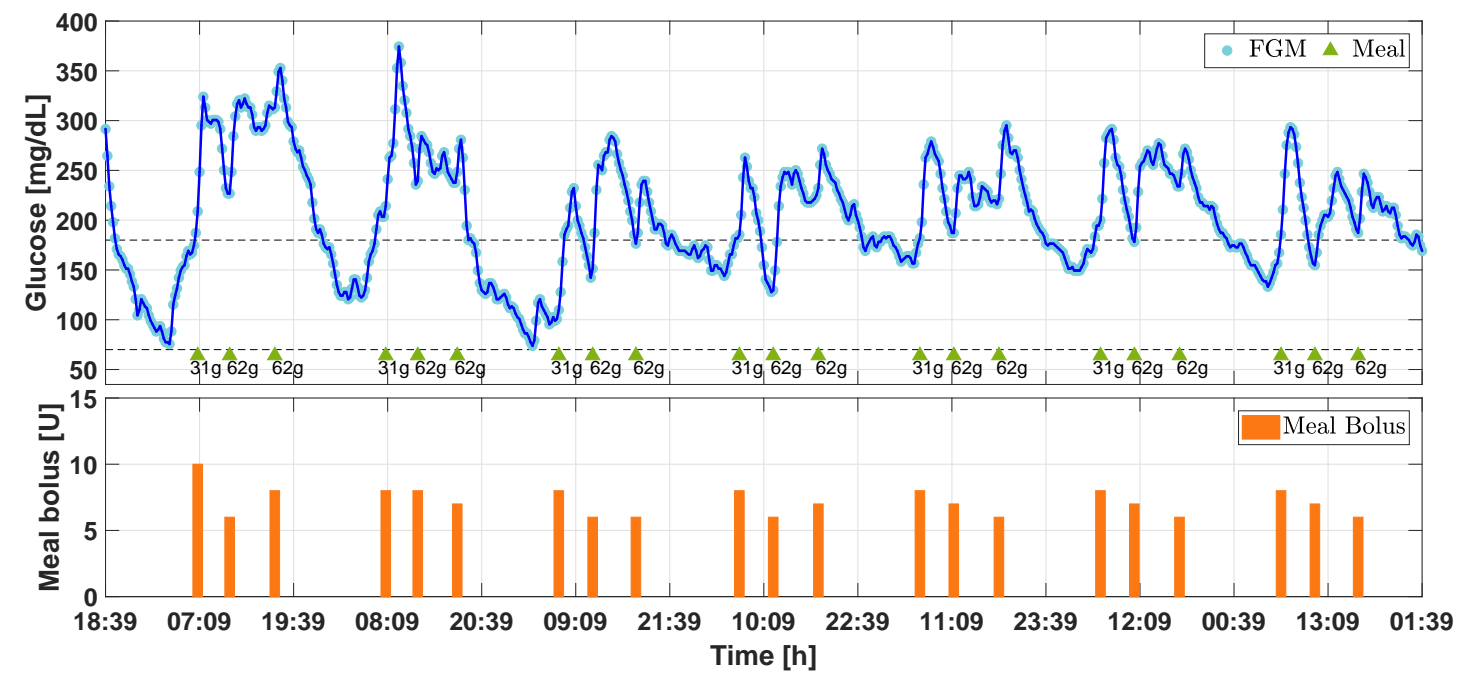

Figure 4: The collection of the clinical data from a T1DM subject. Meals are denoted by green triangles with sizes below them, and the corresponding meal boluses determined by the clinicians are displayed in the second panel.

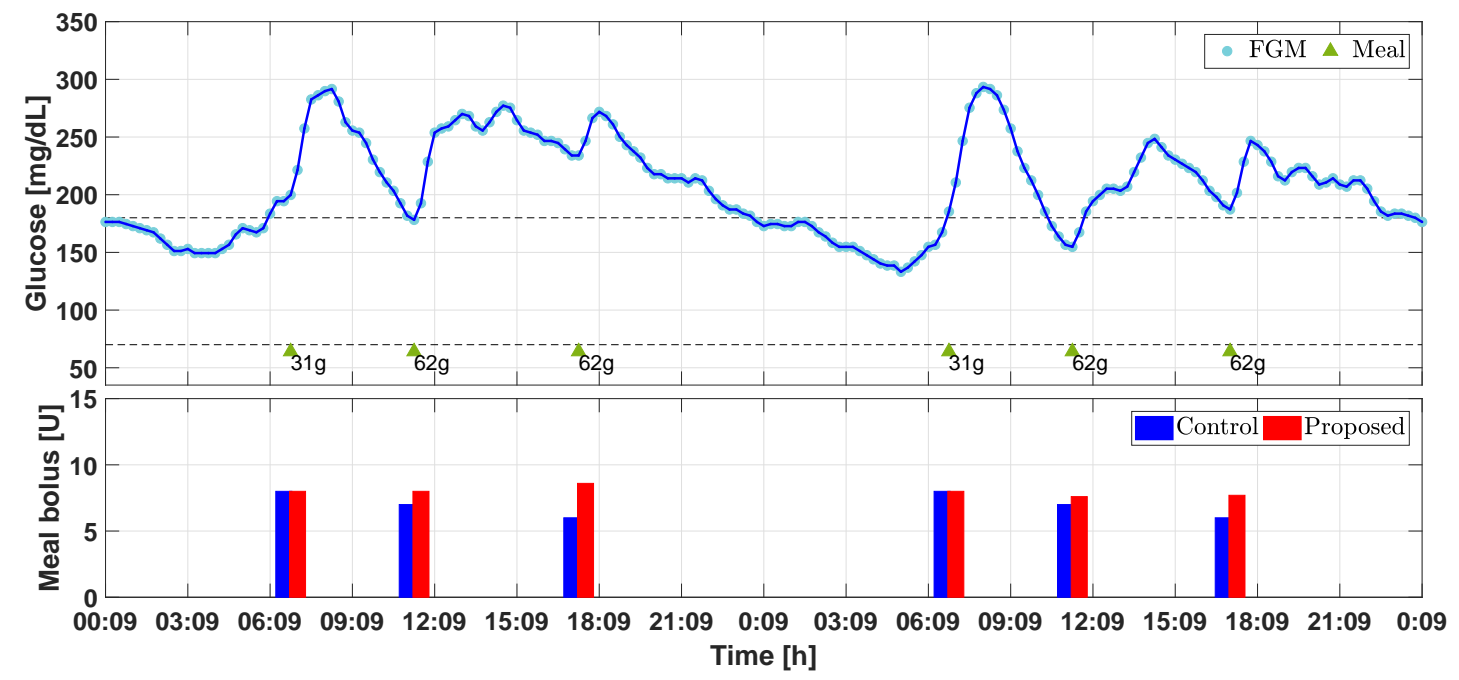

Figure 5: Performance evaluation of the proposed method based on the clinical data. Meals are denoted by green triangles with sizes below them.

\section{References}

[1] Diabetes Control and Complications Trial Research Group, The effect of intensive treatment of diabetes on the development and progression of long-term complications in insulin-dependent diabetes mellitus, N. Engl. J. Med. 1993 (329) (1993) 977-986.

[2] A. Schiffrin, M. M. Belmonte, Multiple daily self-glucose monitoring: its essential role in long-term glucose control in insulin-dependent diabetic patients treated with pump and multiple subcutaneous injections, Diabetes Care 5 (5) (1982) 479-484.

[3] F. J. Doyle III, L. M. Huyett, J. B. Lee, H. C. Zisser, E. Dassau, Closedloop artificial pancreas systems: engineering the algorithms, Diabetes care 37 (5) (2014) 1191-1197.

[4] A. Cinar, Artificial pancreas systems: An introduction to the special issue, IEEE Control Syst. Mag. 38 (1) (2018) 26-29.

[5] D. Boiroux, A. K. Duun-Henriksen, S. Schmidt, K. Nørgaard, N. K. Poulsen, H. Madsen, J. B. Jørgensen, Adaptive control in an artificial pancreas for people with type 1 diabetes, Control Eng. Pract. 58 (2017) $332-342$.

[6] G. P. Incremona, M. Messori, C. Toffanin, C. Cobelli, L. Magni, Model predictive control with integral action for artificial pancreas, Control Eng. Pract. 77 (2018) 86 - 94.

[7] A. El Fathi, M. Raef Smaoui, V. Gingras, B. Boulet, A. Haidar, The artificial pancreas and meal control: An overview of postprandial glucose regulation in type 1 diabetes, IEEE Control Syst. 38 (1) (2018) 67-85.

[8] C. Owens, H. Zisser, L. Jovanovic, B. Srinivasan, D. Bonvin, F. J. Doyle III, Run-to-run control of blood glucose concentrations for people with type 1 diabetes mellitus, IEEE Trans. Biomed. Eng. 53 (6) (2006) 9961005.

[9] M. Schiavon, C. Dalla Man, C. Cobelli, Insulin sensitivity index-based optimization of insulin to carbohydrate ratio in silico study shows efficacious protection against hypoglycemic events caused by suboptimal therapy, Diabetes Technol. Ther. 20 (2) (2018) 98-105.

[10] P. Herrero, P. Pesl, M. Reddy, N. Oliver, P. Georgiou, C. Toumazou, Advanced insulin bolus advisor based on run-to-run control and case-based reasoning, IEEE J. Biomed. Health Informat. 19 (3) (2015) 1087-1096.

[11] F. Torrent-Fontbona, B. Lopez, Personalized adaptive CBR bolus recommender system for type 1 diabetes, IEEE J. Biomed. Health Informat. 23 (1) (2019) 387-394.

[12] S. W. Liu, H. P. Huang, C. H. Lin, I. L. Chien, Fuzzy-logic-based super- 
visor of insulin bolus delivery for patients with type 1 diabetes mellitus, Ind. Eng. Chem. Res. 52 (4) (2013) 1678-1690.

[13] Q. Sun, M. Jankovic, J. Budzinski, B. Moore, P. DIem, C. Stettler, S. Mougiakakou, A dual mode adaptive basal-bolus advisor based on reinforcement learning, IEEE J. Biomed. Health Informat. 23 (6) (2019) 2633-2641.

[14] K. Turksoy, I. Hajizadeh, S. Samadi, J. Feng, M. Sevil, M. Park, L. Quinn, E. Littlejohn, A. Cinar, Real-time insulin bolusing for unannounced meals with artificial pancreas, Control Eng. Pract. 59 (2017) 159 - 164.

[15] C. Toffanin, R. Visentin, M. Messori, F. D. Palma, L. Magni, C. Cobelli, Toward a run-to-run adaptive artificial pancreas: In silico results, IEEE Trans. Biomed. Eng. 65 (3) (2018) 479-488.

[16] D. Shi, E. Dassau, F. J. Doyle III, Multivariate learning framework for long-term adaptation in the artificial pancreas, Bioeng. Transl. Med. (2018) 1-14.

[17] A. Z. Woldaregay, E. Arsand, S. Walderhaug, D. Albers, L. Mamykina, T. Botsis, G. Hartvigsen, Data-driven modeling and prediction of blood glucose dynamics: Machine learning applications in type 1 diabetes, Artif. Intell. Med. 98 (Jul.) (2019) 109-134.

[18] H. M. Romero-Ugalde, M. Garnotel, M. Doron, P. Jallon, G. Charpentier, S. Franc, E. Huneker, C. Simon, S. Bonnet, ARX model for interstitial glucose prediction during and after physical activities, Control Eng. Pract. 90 (2019) $321-330$.

[19] X. Yu, K. Turksoy, M. Rashid, J. Feng, N. Hobbs, I. Hajizadeh, S. Samadi, M. Sevil, C. Lazaro, Z. Maloney, E. Littlejohn, L. Quinn, A. Cinar, Model-fusion-based online glucose concentration predictions in people with type 1 diabetes, Control Eng. Pract. 71 (2018) 129 - 141.

[20] E. Georga, V. Protopappas, D. Polyzos, D. Fotiadis, Evaluation of shortterm predictors of glucose concentration in type 1 diabetes combining feature ranking with regression models, Med. Biol. Eng. Comput. 53 (12) (2015) 1305-1318.

[21] E. Montaser, J.-L. Diez, P. Rossetti, M. Rashid, A. Cinar, J. Bondia, Seasonal local models for glucose prediction in type 1 diabetes, IEEE J. Biomed. Health Informat. 24 (7) (2020) 2064-2072.

[22] M. Deisenroth, D. Fox, C. Rasmussen, Gaussian processes for data-efficient learning in robotics and control, IEEE Trans. Pattern Anal. Mach. Intell. 37 (2) (2015) 408-423.

[23] A. Jain, T. X. Nghiem, M. Morari, R. Mangharam, Learning and control using gaussian processes, In Proceedings of the 9th ACM/IEEE International Conference on Cyber-Physical Systems (ICCPS) (2018).

[24] X. Yang, J. Maciejowski, Risk-sensitive model predictive control with gaussian process models, IFAC-PapersOnLine 48 (28) (2015) 374-379.

[25] Y. Pan, G. Boutselis, E. Theodorou, Efficient reinforcement learning via probabilistic trajectory optimization, IEEE Trans. Neural Netw. Learn. Syst. 29 (11) (2018) 5459-5474.

[26] B. Shahriari, K. Swersky, Z. Wang, R. P. Adams, N. De Freitas, Taking the human out of the loop: A review of bayesian optimization, Proceedings of the IEEE 104 (1) (2015) 148-175.

[27] R. Gillis, C. C. Palerm, H. Zisser, L. Jovanovic, D. E. Seborg, F. J. Doyle III., Glucose estimation and prediction through meal responses using ambulatory subject data for advisory mode model predictive control, J. Diabetes Sci. Technol. 1 (6) (2007) 825-833.

[28] S. Roberts, M. Osborne, M. Ebden, S. Reece, N. Gibson, S. Aigrain, Gaussian processes for time-series modelling, Philos. Trans. Roy. Soc. London A, Math. Phys. Sci. 371 (1984) (2013) 1-14.

[29] C. E. Rasmussen, C. K. I. Williams, Gaussian Processes for Machine Learning, the MIT Press, 2006.

[30] L. P. R. Bergman, C. Cobelli, Physiologic evaluation of factors controlling glucose tolerance in man, The Journal of Clinical Investigation 68 (6) (1981) 1456-1467.

[31] R. Hovorka, V. Canonico, L. J. Chassin, U. Haueter, M. Massi-Benedetti, M. O. Federici, T. R. Pieber, H. C. Schaller, L. Schaupp, T. Vering, Nonlinear model predictive control of glucose concentration in subjects with type 1 diabetes, Physiol. Meas. 25 (4) (2004) 905-920.

[32] C. Dalla Man, R. A. Rizza, C. Cobelli, Meal simulation model of the glucose-insulin system, IEEE Trans. Biomed. Eng. 54 (10) (2007) 17401749 .

[33] R. Gondhalekar, E. Dassau, F. J. Doyle III, Periodic zone-MPC with asymmetric costs for outpatient-ready safety of an artificial pancreas to treat type 1 diabetes, Automatica 71 (2016) 237-246.

[34] J. B. Lee, E. Dassau, R. Gondhalekar, D. E. Seborg, J. E. Pinsker, F. J.
Doyle III, Enhanced model predictive control (eMPC) strategy for automated glucose control, Ind. Eng. Chem. Res. 55 (46) (2016) 1185711868.

[35] P. Whittle, J. Kuhn, A hamiltonian formulation of risk-sensitive Linear/Quadratic/Gaussian control, Int. J. Control 43 (1) (1986) 1-12.

[36] D. R. Jones, M. Schonlau, W. J. Welch, Efficient global optimization of expensive black-box functions, J. Glob. Optim. 13 (4) (1998) 455-492.

[37] R. Gondhalekar, E. Dassau, F. J. Doyle III, Velocity-weighting \& velocity-penalty MPC of an artificial pancreas: Improved safety \& performance, Automatica 91 (2018) 105 - 117.

[38] C. Dalla Man, F. Micheletto, D. Lv, M. Breton, B. Kovatchev, C. Cobelli, The UVA/Padova type 1 diabetes simulator: new features, J. Diabetes Sci. Technol. 8 (1) (2014) 26-34. 